\title{
GLAD!
}

Revue sur le langage, le genre, les sexualités

\section{Institution familiale et injonction à} l'hétérosexualité à Dakar

À propos de la réputation masculine d' " aimer les femmes »

Family Institution and the Injunction to Heterosexuality in Dakar : About the

Masculine Reputation of "Loving Women"

\section{Nicolas Faynot}

\section{OpenEdition}

\section{Journals}

Édition électronique

URL : http://journals.openedition.org/glad/747

DOI : $10.4000 /$ glad.747

ISSN : 2551-0819

Éditeur

Association GSL

\section{Référence électronique}

Nicolas Faynot, «Institution familiale et injonction à l'hétérosexualité à Dakar », GLAD! [En ligne], 03 | 2017, mis en ligne le 10 décembre 2017, consulté le 20 janvier 2021. URL : http:// journals.openedition.org/glad/747 ; DOI : https://doi.org/10.4000/glad.747

Ce document a été généré automatiquement le 20 janvier 2021.

\section{(c) (i) $(9)$}

La revue GLAD! est mise à disposition selon les termes de la Licence Creative Commons Attribution Pas d'Utilisation Commerciale - Pas de Modification 4.0 International. 


\title{
Institution familiale et injonction à l'hétérosexualité à Dakar
}

\author{
À propos de la réputation masculine d' « aimer les femmes » \\ Family Institution and the Injunction to Heterosexuality in Dakar: About the \\ Masculine Reputation of "Loving Women"
}

Nicolas Faynot

1 La réputation du goor gu bëgg jigeen, qui en langue wolof signifie littéralement l'« homme qui aime les femmes ", est ambiguë dans le sens où elle peut être tout aussi valorisante que dévalorisante. Quoi qu'il en soit, elle concerne directement la sexualisation de l'identité de l'homme ainsi désigné à travers une mise en perspective de sa virilité. Cette contribution souhaite, en se saisissant de cette réputation, s'intéresser aux manifestations discursives qui entourent les normes hétérosexuelles, lorsqu'elles émanent des membres d'une institution familiale sénégalaise pour être dirigées vers ses jeunes hommes. J'insisterai particulièrement sur les injonctions à l'hétérosexualité, donc à l'hétéromasculinité ${ }^{1}$, contenues dans ces manifestations. Par ce fait, je souhaite m'ancrer dans les études émanant de diverses disciplines qui, tout en portant sur différentes chronicités et zones géographiques, ont permis d'asseoir les questions concernant la construction des masculinités dans un débat contemporain, et de lancer un appel quant à la nécessité de les poursuivre et de les enrichir. Dans cette logique, l'étude des masculinités au sein de contextes familiaux semble d'une importance considérable, puisqu'elle permet de se rendre compte combien l'instance familiale prend part à la reproduction de la domination masculine, par quels mécanismes elle tend à la reproduire et quelle place elle occupe dans la fabrication des identités sexuelles (Bourdieu 2002 ; Sohn 2009 ; Godelier 2004).

2 La complexité et la relativité de l'image du goor gu bëgg jigeen semblent particulièrement appropriées pour interroger les masculinités, leurs dynamiques et les mécanismes d'hétérosexualisation qui émanent d'une institution sociale sénégalaise. Avant de commencer cette analyse, il me faudra introduire le cadre de cette recherche et revenir sur les choix méthodologiques qui ont été faits. Ces informations apportées, 
on pourra se concentrer sur les critères qui tendent à justifier l'attribution de la réputation de goor gu bëgg jigeen. La trajectoire d'un jeune homme sera étudiée afin d'envisager concrètement par quels moyens cette assignation opère, tout en reconnaissant qu'il y a des "logiques sociales à l'œuvre dans la production et la diffusion des dispositifs de réputation " (Beuscart et al $2015: 6$ ). Cette trajectoire sera par la suite comparée à celle d'un autre homme pour envisager la non-attribution de cette réputation et ses implications. Je montrerai comment l'image en question revêt une forme d'injonction sociale de virilité, ce qui permettra de revenir sur certains de ses mécanismes. J'introduirai le fait, à travers deux autres trajectoires masculines, que cette réputation puisse dans certaines de ses configurations être dévalorisante, avec l'idée sous-jacente qu'à travers elle, il existe un jugement social des mesures du désir. Pour finir, j'envisagerai la notion de réputation en tant que phénomène langagier et comme un dispositif de jugement, permettant de montrer les tensions existantes entre virilité et obligations masculines. Dans ce cadre, il s'avère intéressant de constater dans quelle mesure cette assignation peut entraîner des hommes à dénoncer les formes que prend l'injonction à l'hétéromasculinité.

\section{Cadre empirique et théorique de la recherche}

3 C'est dans le cadre d'un travail de thèse en anthropologie s'intéressant aux trajectoires sentimentales prémaritales des hommes dakarois que les données présentées dans cet article ont été collectées entre 2013 et $2016^{2}$. Afin de m'intéresser à la production d'une injonction à l'hétérosexualité, j'ai opté pour la restitution des trajectoires de quatre jeunes hommes de cette famille qui présentent donc des caractéristiques communes: ils appartiennent à la même ethnie (Wolof), à la même confession religieuse (musulmane de confrérie mouride) et ne sont pas mariés ${ }^{3}$. Ces choix méthodologiques se justifient à plusieurs égards. Il m'importe tout d'abord de m'attarder sur des cas particuliers dans un souci de précision empirique. De plus, prendre pour assise une seule unité familiale limite le nombre de relations interpersonnelles à prendre en compte. Ensuite, le choix d'une analyse en contexte familial permet de porter une attention particulière à ce cadre de socialisation et aux liens ambigus qu'il entretient avec le mécanisme réputationnel dont il est question. Enfin, ces quatre profils masculins étant hétérogènes, leurs trajectoires me semblent refléter une large dimension des catégorisations différentes et complémentaires de figures du goor gu bëgg jigeen. Les hommes en question sont deux frères de mêmes père et mère, et deux de leurs cousins par filiation utérine qui sont eux aussi frères de mêmes père et mère.

Il m'a fallu investir des espaces ${ }^{4}$ pour les besoins de cette enquête de manière régulière sur une temporalité s'étalant sur trois ans. Ainsi, j'ai pu partager de nombreux moments informels avec une grande partie des membres de cette famille. Les données présentées dans cet article émanent de méthodes de recueil variées et complémentaires. Ainsi, j'ai pu observer de multiples interactions entre ces hommes et des membres de leur famille, qui ont eu lieu parfois en présence d'une de leurs petites amies. Ensuite, des entretiens semi-directifs ont été menés avec ces hommes ${ }^{5}$, visant à recueillir des récits sexuels (Giami 2000) afin de tenter de comprendre au mieux leurs trajectoires sentimentales. Ils ont été complétés par des entretiens non directifs et semi-directifs conduits auprès de leurs parents, de leurs oncles et tantes, d'une partie de leurs cousins / cousines et frères / sœurs. Enfin, boire le thé avec ces hommes en 
compagnie de leurs amis m'a permis d'avoir accès à des conversations au cours desquelles ils reviennent souvent sur leurs parcours sentimentaux.

5 L'approche adoptée porte une attention particulière aux discours de ces hommes ainsi qu'à ceux qui sont émis sur eux par les membres de leur famille. Puisqu'il sera question d'une réputation qui semble se manifester et se négocier surtout par des actes langagiers, procéder à une analyse de ces discours se révèle incontournable ${ }^{6}$. Il convient alors de préciser que, afin de répondre aux objectifs de cet article, j'insisterai surtout sur la contextualisation de ces activités discursives (Hymes 1996), en mettant au jour les positions des locuteurs en termes de relations sociales et de pouvoir. Ce que je chercherai surtout à travers cet ancrage sera l'exploration des dimensions interactionnelles et performatives des pratiques langagières (Masquelier 2005), afin de mieux saisir les enjeux entourant le dispositif réputationnel analysé.

\section{Les critères d'évaluation de la réputation}

6 Tapha a 18 ans $^{7}$. Il est scolarisé dans un lycée du secteur. Comme un grand nombre de ses camarades de classe, il a plusieurs petites amies et passe des heures à converser au téléphone avec elles. Dès qu'il rentre du lycée, il monte sur la terrasse et les appelle. Lorsque ses aînés l'envoient faire des commissions, il tarde souvent à se mettre en route, puisque cela l'oblige à abréger ses conversations téléphoniques. Un dimanche de mars 2015, l'une de ses petites amies, Khadi, vient lui rendre visite. Elle est invitée à s'asseoir dans le salon. Tout en regardant une série télévisée, Tapha et Khadi conversent et se demandent réciproquement des nouvelles de leur famille. Vingt minutes plus tard, ils s'éclipsent dans une chambre dont ils ne ferment pas la porte, seul un rideau opaque est tiré. Dix minutes plus tard ils sortent, et Tapha la raccompagne. Il montera alors sur la terrasse pour appeler une autre de ses petites amies. Après quarante minutes de conversation, il appellera Khadi et restera une demiheure au téléphone avec elle. Dans le salon, on parle de lui, sa mère s'adresse à sa bellesœur dans ces termes: "Xoolal sama dom, ki dafa bëgg jigeen » (Regarde mon fils, lui il aime les femmes). Une des tantes confirme et précise qu'il est comme son grand frère, qu'il aime les filles. Suite à cet échange, un oncle de Tapha me confie :

Tu as vu la mère de celui-là [Tapha], comment elle bombe le torse ! Elle est tellement fière.

7 Cette description semble particulièrement renseigner sur certains critères qui tendent à l'évaluation du fait que Tapha "aime les femmes». Les membres de sa famille ont établi la déduction suivante : si Tapha est présent dans l'espace domestique et qu'il ne fait pas ses devoirs, il téléphone forcément à une jeune femme. Pour ses ainés familiaux, c'est un indice manifeste du fait qu'il «aime les femmes ». Un autre aspect revêt également une valeur de preuve quant à la qualification de la réputation du goor gu bëgg jigeen : le fait que plusieurs femmes de son âge viennent rendre visite à Tapha. La visibilité des relations qu'il entretient est en effet mise en lumière à travers ces visites. Lorsqu'il est en déplacement dans le quartier, Tapha n'en rend pas forcément compte à ses aînés. A priori donc, il n'y a pas de contrôle social concernant ses fréquentations en dehors de la maison familiale. Ce sont alors les visites des petites amies de Tapha qui rendent concrètement visibles ses relations amoureuses. Un 
troisième critère semble présent dans l'évaluation de la réputation, mais dans une moindre mesure, celui qui se rapporte au regard.

Quand tu jettes un coup d'œil aux femmes qui passent devant toi, tu regardes leurs formes, tu les regardes, parce que tu les trouves jolies. Si quelqu'un te voit faire ça, il dira que toi, «tu aimes les femmes. (Un cousin de Tapha, 21 ans, 2015).

8 L'orientation du regard confirmerait ainsi une attirance sensuelle envers les corps féminins, ce qui aux dires des aînés de Tapha serait manifestement son cas. Ces trois critères témoigneraient du fait que Tapha puisse être considéré comme "aimant les femmes ». Mis bout à bout, ils confirment la réputation dont il est détenteur. L'évaluation de celle-ci est bien sûr contestable dans la mesure où elle repose sur des critères de "preuves indirectes " (Emler 2013). Elle tient ainsi en grande partie à sa visibilité, au-delà de son évaluation objective. Ce qui semble ici particulièrement intéressant tient au fait que cette dernière s'ancre sur des manifestations diverses qui sont traduites comme des confirmations d'une attirance. On retrouve ainsi deux des caractéristiques de la réputation telle que la définit P. Boistel $(2014: 212-213)$ en s'appuyant sur des analyses de C.Fombrun (1996): le fait qu'elle soit basée sur des perceptions et qu'elle représente une uniformité de perceptions individuelles.

Chez les Wolofs, la réussite de l'éducation d'un enfant est portée au crédit de la mère. L'expression ndey-ju-liggééy (une femme qui a travaillé) témoigne de cette réalité. On dira d'un enfant qui est bien éduqué ou qui a une "bonne situation » que sa mère a «bien travaillé » (Diop 2012: 23; Gning 2013 : 27). On comprend alors le sentiment de fierté de la mère de Tapha, par rapport au comportement de son fils, ce dont témoigne son oncle en exprimant qu'elle " bombe le torse ». À l'instar de la plupart des sociétés, la société wolof se fonde sur la différenciation asymétrique des sexes et l'hétéronormativité ${ }^{8}$ (Gning 2013 : 25). Dans ce contexte, être estimé comme quelqu'un qui « aime les femmes » est valorisant. Si certaines femmes de la famille parlent de Tapha en son absence, elles ironisent sur le fait qu'il est toujours occupé à séduire. Le registre employé n'est pas celui de la moquerie: le rire est suscité par une certaine forme de pudeur, en particulier lorsque le sujet est évoqué dans un cadre transgénérationnel et mixte. Les taquineries sont souvent répétées et assurent verbalement une reconnaissance des qualités hétérosexuelles prêtées à Tapha. Il s'agit dès lors de comprendre que l'étiquette accolée à ce dernier par les membres de sa famille « a un pouvoir performatif immédiat: au moment où elle est prononcée et validée, elle rend réelle, aux yeux de son public, l'attitude qu'elle décrit [...]» (Clair $2008: 82$ ).

10 Incontestablement, par son intérêt pour les femmes, Tapha montre qu'il est en train de devenir un homme. L'attrait qu'il manifeste et l'investissement subjectif temporel qu'il accorde à des jeunes femmes peuvent alors être significatifs sans pour autant être jugés comme « anormaux » du fait de son jeune âge. Il a 18 ans et a déjà prouvé aux membres de sa famille qu'il était apprécié par des jeunes femmes. Il parcourt ainsi un " processus » qui « conduit l'adolescent à l'âge d'homme » (Sohn 2009: 8), et qui est à cet égard socialement valorisé. On se trouve dans un registre de virilité ostentatoire, où les paroles et les actes de Tapha apparaissent comme des preuves de ses capacités, considérées localement comme viriles. Ce qui fait sa réputation, c'est autant d'aimer les femmes que d'être apprécié par elles. Son manque de discrétion dans ses démonstrations amoureuses pourrait s'expliquer par le fait qu'il cherche justement à 
rendre visible son aptitude à plaire et à entretenir différentes relations en même temps, ce qui lui permettrait de jouir des aspects positifs de cette réputation. Il est nécessaire de prendre en considération le fait que le désir d'une "bonne réputation » peut « inspirer l'action » d'un individu (Elster $2013: 29$ ).

\section{L'injonction sociale à la virilité}

11 Les aspects du comportement de Tapha dont il a été question poussent sa famille à le qualifier de jeune homme qui «aime les femmes». Ainsi, qu'il soit porteur de cette réputation est signifié à maintes occasions par les membres de sa famille, en particulier par des actes langagiers, comme le montrent les exemples mentionnés. A contrario, ce n'est pas le cas d'Amar, 20 ans, le cousin de Tapha avec qui il partage la même chambre. Déscolarisé à l'âge de 14 ans, il consacre une grande partie de ses journées à la pratique du football. Il s'entraîne tous les jours depuis quatre ans et espère devenir joueur professionnel. Parce qu'il tente de se consacrer exclusivement au sport, il fait savoir à son entourage familial qu'il ne cherche pas de petite amie. Selon lui, concilier ses espoirs professionnels, qui lui demandent un entraînement physique intensif, au fait d'avoir des petites amies, semble irréalisable :

J'ai déjà eu des copines, mais je n'en veux plus. Ça te fatigue trop. Tu n'as pas la tête à l'entraînement, tu penses tout le temps à elles. Et si tu fais de la sexualité avec elles, après tu es tellement fatigué. Il faut faire des sacrifices. Quand je serai célèbre In Shaa Allah, là, j'aurais le temps pour les filles. (2014)

12 Amar dit refuser engagement sentimental et activité sexuelle parce qu'il se les représente comme nuisibles à son potentiel sportif et ne servant pas l'objectif prioritaire qu'il s'est fixé'.

13 L'observation des comportements relationnels amoureux dans le quartier des Parcelles Assainies me pousse à revenir sur une tendance, révélée par le suivi d'une trentaine de jeunes personnes investies dans des relations de couple, qui veut que toute relation sentimentale entraîne pour les deux protagonistes un investissement temporel conséquent. C'est un critère permettant à chacun des partenaires d'évaluer la force de l'engagement dans la relation ${ }^{10}$. L'investissement temporel apparaît comme un synonyme d'attachement, ce qui implique qu'il soit requis. Amar préfère donc ne pas se permettre d'entreprendre une ou plusieurs relations, et une des raisons apparentes de ce choix porte sur la dimension temporelle qui est étroitement associée aux modalités amoureuses. Ses aînés familiaux n'hésitent pas à se moquer de lui à ce sujet et en parlent souvent en ces termes :

Amar n'aime pas les filles. Il n'aime que son ballon. Sa copine, c'est son ballon. (Une tante d'Amar, 48 ans, 2016)

14 Les enfants de la famille reproduisent ensemble ce genre de moqueries, ce qui les fait beaucoup rire. Lorsque l'un d'eux s'empare d'un ballon, il n'est pas rare de le voir s'approcher d'Amar en le lui tendant et en lui disant : «Regarde ta copine !» En février 2014, alors qu'il était assis dans la rue devant la porte de sa maison, un de ses oncles (49 ans) le voit regarder un groupe de lycéennes qui rentrait de classe, et lui dit :

Pourquoi tu les regardes? Toi, tu n'aimes que ton ballon. Tu ne sais rien! 
contenterait de regarder les jeunes femmes de son âge sans oser interagir avec elles ne pourrait pas être considéré comme un "homme qui aime les femmes». Même si ce regard peut témoigner d'une attirance envers l'autre sexe, il ne semble pas à lui seul un critère permettant de se voir attribuer cette réputation ${ }^{11}$. Celle-ci n'est pas pensée comme liée uniquement à la passivité d'un regard, au ressenti d'un attrait, mais à une mise en acte, à sa concrétisation. C'est par la démonstration de cette attraction et une identification claire envers les femmes séduites que la réputation prend racine, d'où le postulat suivant: cet étiquetage nécessite des preuves en termes de capacités de conquête. De son côté, Amar exprime et montre qu'il rejette momentanément séduction et relations sentimentales, alors s'il lui arrive de « regarder » des femmes, ce regard ne sera pas considéré comme la preuve d'une attirance. Pour Amar, comme pour Tapha, il y a donc réception des signes de l'attirance de la part de tiers, puis interprétation, et c'est cette dernière qui agira positivement ou négativement quant à la validation de cette réputation.

17 Parce qu'il ne montre pas suffisamment de signes d'un certain nombre de capacités attendues chez un homme de son âge, Amar essuie quotidiennement des moqueries de la part de membres de sa famille d'âge et de sexe différents, moqueries qui s'échafaudent autour de la remise en cause d'une qualité perçue comme essentielle de sa masculinité : son orientation sexuelle. On ne peut pas voir dans son ballon de football un fétichisme sexuel dans un sens freudien, puisqu'il n'y a pas remplacement de l'objet sexuel «normal » par un autre objet en relation avec lui (Freud 2012: 83). Toutefois, la désignation d'une attirance envers un objet semble bien présente, et jugée comme relevant d'un critère sexuel, car elle est pensée comme se faisant au détriment de l'attirance pour l'autre sexe. Les projets de carrière d'Amar le poussent à effectuer un choix dans les registres amoureux et sexuel, mais ne sont pas entendus par les membres de sa famille comme une justification valable de son abstinence ${ }^{12}$. Ce qu'ils retiennent tient au non-souhait et à la non-capacité d'Amar de séduire et d'entrer en relation avec des femmes. Cela soulignerait pour eux une « déviance homosexuelle ou asexuelle» :

S'il n'aime pas les femmes, c'est qu'il aime les hommes. (Une sœur d'Amar, 24 ans, 2016)

18 T. K. Biaya met en avant dans ses travaux que les familles dakaroises ne s'inquiètent pas de l'«inertie sexuelle» de leurs «jeunes mâles» (2001:83). Le cas d'Amar tend à montrer sinon le contraire, du moins qu'une partie de sa famille peut s'en soucier.

19 L'injonction sociale à la virilité, telle qu'elle se manifeste au Sénégal en particulier envers les jeunes hommes, entraîne une incompréhension dans le cas où un homme refuse une relation sexuelle avec une femme. Cela s'applique aussi par extension si celui-ci refuse d'entrer dans une relation amoureuse ${ }^{13}$. "L'homme est naturellement polygame », comme se plaît à le dire un grand nombre de mes interlocuteurs masculins, les jeunes hommes non mariés n'ayant que peu d'injonctions à une exclusivité amoureuse et sexuelle. Je ne m'étendrai pas sur les justifications culturelles et religieuses qui sous-tendent ce propos, ce qui importe est cette obligation sociale de

GLAD!, 03 | 2017 
virilité et les manières dont elle s'exerce. À ce stade de la réflexion, il semble possible d'admettre que la réputation du goor gi bëgg jigeen s'attache en particulier aux amours plurielles et mette en avant non seulement une orientation sexuelle affirmée, mais aussi des capacités relationnelles ${ }^{14}$. Elle encourage en effet la prise d'initiative, le passage à l'acte dans la séduction, et c'est précisément en cela que l'attirance envers les femmes ne suffit pas.

Les femmes maintenant, elles sont plus difficiles qu'avant. Elles ne se laissent pas faire, elles veulent tout contrôler. Elles sont fortes. Il faut que les jeunes garçons les connaissent bien pour les maîtriser, sinon on dit que ta femme, elle ne te respecte pas, et ça, c'est pas bon! (2014)

Le vocabulaire mobilisé par la mère d'Amar, d'une cinquantaine d'années, n'est pas équivoque. Outre le fait qu'il met en avant le poids des rapports de genre et une volonté de reproduction de ceux-ci, il indique aussi que c'est par l'expérience qu'un homme devient capable de «contrôler». Ne pas «connaître» et "refuser» des femmes, comme c'est apparemment le cas de son fils, questionne par extension sa capacité à prendre épouse et à se faire respecter en tant qu'époux.

Le contraire de bëgg jigeen (aimer les femmes), c'est xamul jigeen (il ne connaît pas les femmes) ou dafa bagñe jigeen (il refuse les femmes). C'est grave si on dit ça d'un homme, ça veut dire qu'il est homosexuel. (Tapha, 2013)

21 Le fait de dire «non» aux femmes est jugé d'autant plus grave qu'un doute est alors permis sur l'hétérosexualité dans un cadre culturel caractérisé par une "norme homophobe affirmée » (Salomon 2009: 154). Ne "pas connaître les femmes» montre qu'un homme n'est pas apte à entreprendre des relations du fait de sa méconnaissance des arts de la séduction ou tout simplement de son incompétence.

\section{Les mesures du désir}

22 Ali est un des frères aînés d'Amar. Il a fait le choix il y a quatre ans de quitter la résidence familiale pour s'installer en centre-ville dans une chambre d'un appartement locatif qu'il partage avec un de ses cousins. Il a pris cette décision afin de se libérer quelque peu de l'emprise de sa famille, qu'il jugeait trop forte, et pour tenter de faire $\mathrm{du}$ « business » en ville. Ce choix de l'habitat demeure une source de tension entre Ali et sa famille, puisque celle-ci lui reproche son manque de considération et de solidarité. Après avoir déménagé, il aurait totalement délaissé les membres de sa famille en ne participant plus aux dépenses quotidiennes. Ses proches lui tiennent rigueur de ce manque d'investissement, et déplorent de ne pas recevoir d'apport financier de sa part. Ali mène une vie de noctambule composée de sorties dans des bars et en boîte de nuit et il n'hésite pas à le faire savoir à qui veut l'entendre. À ce titre, il insiste tout particulièrement sur la narration de ses multiples conquêtes amoureuses et sexuelles, tout en se vantant d'avoir des petites amies dans tous les quartiers de Dakar. Ainsi lorsque son oncle (53 ans, 2014) dit de lui « Ali bëgg na jigeen trop ${ }^{15}$ " (Ali aime trop les femmes), il exprime que ce dernier aime «trop » les femmes aux dépens de sa famille. Cette énonciation met l'accent, dans cette évaluation du désir, sur le fait qu'il les aime de manière démesurée. Cet excès de désir pour les femmes est alors compris comme étant la source de la négligence d'Ali envers sa famille ${ }^{16}$. Attané nous dit à ce propos, en référence au contexte burkinabais, que "l'intérêt personnel ne doit pas supplanter les 
obligations sociales d'entraide vis-à-vis des proches, obligations auxquelles chacun est soumis » (2009: 158). Le comportement d'Ali est loin d'être perçu comme un modèle, un exemple à suivre pour les plus jeunes hommes de la famille, mais plutôt comme un vecteur de honte. Ces reproches touchent également à la notion de piété dans l'éducation religieuse musulmane au Sénégal, qui met un accent fort sur les obligations genrées des hommes (Dimé 2007 ; Vuarin 1990) ${ }^{17}$. Dans le cas particulier d'Ali, c'est bien autour du non-respect d'une valeur masculine et de la rupture avec le communautarisme familial ${ }^{18}$ que tournent les reproches qui lui sont adressés. Le jugement social stigmatisant qui va être émis à l'encontre d'Ali se fonde sur sa supposée obsession sexuelle. Son "désir» est représenté comme "trop important», surtout parce qu'il l'empêche d'exercer un rôle social déterminé, conformément à une solidarité économique exigée des hommes envers leur famille (Antoine et al 1998).

Ce n'est pas autour de cette prérogative que vont s'organiser les reproches liés aux comportements d'Idris. À 33 ans, il est l'aîné des fils de son père. Sa situation de fonctionnaire paraît confortable et il réside à proximité de la maison familiale qu'il a quittée depuis six ans pour louer une petite chambre avec un ami. Cela lui permet d'être présent et de répondre à ses obligations familiales. Puisqu'il est très investi dans le budget et les dépenses de sa famille en participant aux charges financières, il répond à son "devoir de solidarité " (Dimé 2007: 160). Depuis qu'il a 17 ans, Idris est multipartenaire, et depuis lors est considéré comme un «homme qui aime les femmes ». Sa relation la plus longue a duré cinq ans, et s'est interrompue lorsque la jeune femme a décidé d'épouser un autre de ses prétendants. Tous les membres de sa famille, mais aussi les habitants du quartier qui le connaissent, le considèrent comme un dorankat bu mag (un grand dragueur).

Quand une de ses tantes (63 ans) parle de lui, elle tient ces propos:

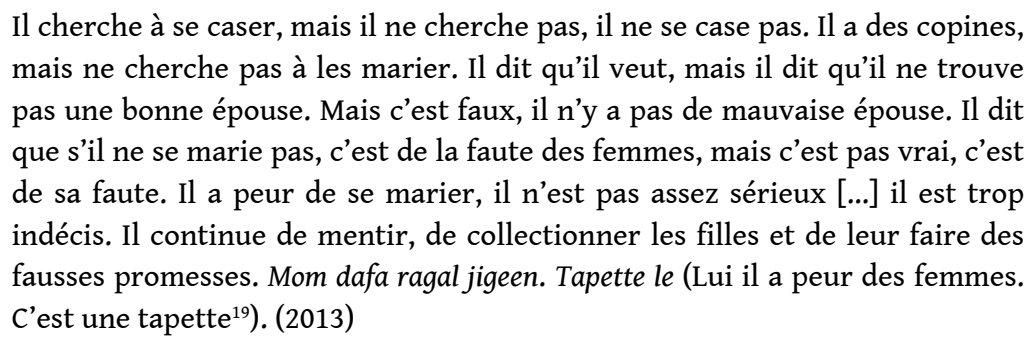

Pour une grande majorité des aînés familiaux d'Idris - et en particulier les femmes -, c'est l'indécision qui caractérise ses comportements en matière de relations amoureuses et c'est ce qui lui est directement reproché. Dans sa fratrie, deux de ses frères cadets sont déjà mariés, et parce qu'il est leur aîné, son indécision est d'autant moins comprise. Son comportement multipartenarial qui ne laisse pas percevoir de perspectives maritales pousse certains à remettre en cause son aptitude à se choisir une seule partenaire et à adopter un comportement jugé responsable. Sa "peur des femmes " n'est pas perçue comme masculine, apparaît comme tout sauf valorisante et sert régulièrement d'explication à une absence de volonté de légitimer une union. Les critiques familiales sont exacerbées par la situation professionnelle d'Idris, qui lui permettrait concrètement de se marier, d'entretenir sa future épouse et les enfants qui naîtraient de cette union. Si sa peur est supposée, c'est aussi parce que les membres de sa famille considèrent qu'il n'arrive pas à "gérer " ses petites amies et les relations qu'il entretient avec elles. Il ne les «maîtriserait » pas assez bien, il n'arriverait pas à 
faire durer ses relations assez longtemps ni sur des bases assez solides et prometteuses pour envisager une union. Il faut replacer l'importance du mariage dans ce contexte bien précis où il est une "étape décisive " - pour les hommes comme pour les femmes - qui, identitairement, entraîne l'accès à une «majorité sociale» (Thoré 1964 : 547-548). Un des frères d'Idris (27 ans) confirme cette hypothèse :

On dit qu'il [Idris] n'est pas fidèle, on dit qu'il est un coureur de jupons. Ça veut dire qu'il n'est pas fidèle à sa famille, qu'il n'est pas fier, parce que sa famille voudrait qu'il soit un leader, un repère pour ses enfants et ses petits frères. Si quelqu'un court derrière les filles, tu le perçois pas comme un modèle. (2016)

Un retour sur ces deux trajectoires de jeunes hommes montre que le « désir » masculin peut être l'objet d'un jugement. La mesure émise par le groupe familial définit implicitement le fait qu'il y aurait de "bonnes » et de "mauvaises » manières de "désirer ", qui se manifestent dans les cas particuliers qui ont été mis en avant en termes d'intensité et de temporalité. Il est donc possible d'approfondir cette réflexion quant à la catégorisation du goor gu bëgg jigeen : pour que cette réputation soit valorisée socialement, il faut « aimer », mais pas « trop », ni « trop longtemps ».

\title{
Réputation et performativité
}

Les données recueillies permettent de mettre au jour que des injonctions hétéronormatives sont émises envers quatre des jeunes hommes de cette famille. C'est sur leurs modalités d'expression que je vais à présent insister. Les travaux d'Austin concernant les actes de langages renseignent sur ce point, en particulier du moment que l'on considère que les types de discours qui ont été mis en avant n'ont pas seulement pour finalité de faire un constat (1991). À bien des égards, les membres de cette famille, lorsqu'ils affirment devant tel ou tel jeune homme qu'il «aime les femmes ", se situent dans un "processus relationnel visant à influencer, orienter, agir sur les autres et sur le monde» (Leimdorfer 2010:4). Il serait alors pertinent de concevoir la réputation comme un acte de langage motivé et performatif (Austin 1991; Clair $2008: 82$ ), dans le sens où il cherche à accomplir une action. L'énoncé en question, situé dans un processus interactionnel, se révèle avoir des effets concrets.

Les propos que peut tenir Ali vont dans ce sens:

\begin{abstract}
Ali beug na jigeen (Ali aime les femmes). Les femmes ici, elles me disent tout le temps ça. Elles me laissent pas tranquille. [...] Elles disent que j'aime trop les femmes. Que je dois pas faire ça. [...] Même si je me marie, les gens ils continueront de parler et dire que j'aime trop les femmes de toute façon. Elles disent: Ali beug na jigeen waye amoul jigeen (Ali aime les femmes mais il n'a pas de femme). (2014)
\end{abstract}

29 Ce pessimisme est imputable à des caractéristiques attribuées à Ali en dépit de sa volonté et de ses qualités individuelles objectives (Becker 1988). Même si la visibilité de ses pratiques amoureuses a contribué à ce qu'il obtienne la réputation en question, cette dernière lui reste accolée et nuit à ses perspectives matrimoniales. Sa réaction sera alors de poursuivre ses pratiques multipartenariales en l'absence de probabilité d'entamer une relation sur des bases différentes, qui ne serait pas entachée de tels présupposés sur sa personne. Amar m'expliquera par ailleurs en 2015 qu'une des 
raisons qui le poussent à ne pas rendre visibles ses relations - car il s'avère qu'il a deux petites amies - tient au fait de ne pas subir les mêmes préjugés qui ont été portés sur Ali à son âge. Le dispositif réputationnel imposerait donc bel et bien des " contraintes " qui s'inscrivent dans la durée (Beuscart et al 2015 : 15). Dans le cas d'Idris, on peut percevoir la manière dont un doute se porte sur la masculinité d'un homme, alors qu'il remplit tous les critères supposés définir a priori une virilité valorisée socialement. On peut ainsi souligner l'aspect «non statique » de la réputation (Chauvin $2013: 141$ ) avec ce passage d'une considération positive à une considération négative : une virilité jugée trop forte peut entrer en contradiction avec les valeurs masculines. Je nuance ainsi les propos de T.K. Biaya, qui soutient que la masculinité se définit par une "sexualité luxurieuse » (2001: 77). Cette étude tend à montrer qu'elle n'en est qu'un aspect, la reconnaissance sociale d'une «bonne» masculinité est relative à des critères plus larges. «La masculinité ainsi entendue se traduit [...] en processus de reconnaissance sociale du sujet » qui passe par « le mariage », donc par une " union hétérocentrée dans un but de procréation » (Fidolini 2017). Cette alliance peut alors être empêchée, tout du moins retardée, par l'incapacité de certains hommes à pouvoir restreindre et maîtriser leur désir des femmes.

La mobilisation de ces différents profils biographiques masculins, en lien avec leur catégorisation ou non comme goor gu bëgg jigeen, a servi de socle afin de présenter différents enjeux en termes de masculinité/virilité. J'ai montré comment cette assignation hétéromasculine est formée par ces enjeux, en mettant en avant la portée prescriptive de ce mécanisme réputationnel. Ce qui semble plausible dans le cadre analysé, et je partage ainsi les opinions de N. Emler, c'est que la réputation serait à voir comme un énoncé de jugement (2013: 91), une manière pour un groupe familial de faire entendre son point de vue sur des manières de se comporter en tant qu'homme. On peut effectivement sentir que c'est un moyen pour des aînés familiaux, pressés que leurs membres se conforment à des normes, de les signifier. Cela passerait par le fait d'encourager les adolescents à se familiariser avec les normes de séduction en vigueur, et les hommes plus âgés à adopter un comportement plus mesuré. Que ce mécanisme fonctionne est une autre question, et j'ai tenté d'en révéler quelques failles. Les particularités du dispositif réputationnel font que les jugements qu'il contribue à faire peser sur un individu s'imposent et échappent en partie au contrôle de celui-ci, d'autant plus lorsqu'ils ne restent pas cantonnés au cercle familial et le débordent rapidement. Cela fut le cas d'Ali et d'Idris, notamment parce qu'ils sont plus âgés qu'Amar et Tapha et qu'ils ont un passif multipartenarial plus important. Les ragots seraient à ce titre des vecteurs de diffusion de présupposés identitaires auxquels on attribue un statut de vérité ${ }^{20}$. Et «le ragot constitue en outre un moyen stratégique pour promouvoir les intérêts des différentes factions ou gérer les réputations et sert donc à exprimer les enjeux de pouvoir» (Bonhomme 2009: 14). Du fait que «la réputation informe sur les autres" (Origgi 2013: 7), dans un climat caractérisé prémaritalement par de fortes suspicions d'infidélité, Ali et Idris portent une réputation rebutante pour les potentielles prétendantes au mariage qu'ils pourraient rencontrer. Quel est l'intérêt, pour les différents membres de la famille, de faire perdurer la réputation d'Ali et d'Idris, alors qu'elle semble les sanctionner et ne pas servir les intérêts supposés de la parentèle?

31 Afin de tenter de répondre à cette question, il s'agit de faire ressortir les modalités particulières de ces situations interactionnelles, pour ainsi dégager les relations 
hiérarchiques dans lesquelles la perpétuation de la réputation prend racine. Si l'on revient sur le fait que c'est entre autres par le ragot que la réputation se propage, sa "circulation au sein du groupe permet de réaffirmer les valeurs communes en stigmatisant les comportements déviants» (Bonhomme 2009: 14). L'aspect de la réputation-sanction est ainsi parfaitement visible dans les propos d'Ali et d'Idris. Ils identifient comme éléments déclencheurs la rupture d'Ali par rapport au principe de solidarité économique institutionnelle, et l'occasion manquée d'Idris d'épouser son expetite amie, qui était très appréciée de ses sœurs et de sa mère. Et selon eux, ce sont surtout les femmes de la famille qui relaieraient le plus d'informations stigmatisantes les concernant. Bien sûr, il faut préciser que ces réponses incriminant principalement les femmes ont été données à un ethnologue homme. Elles font en outre des femmes les vecteurs principaux de la circulation du ragot sans prendre en compte le fait que cellesci, étant beaucoup plus souvent présentes dans l'enceinte familiale et celle du quartier, ont plus l'occasion de les véhiculer.

Moi si j'avais été à sa place [Idris], j'aurais fait pareil. Aujourd'hui, on est moins poussés à se marier que dans le temps. C'est un homme, et il profite.

C'est normal. (Le père d'Idris, 69 ans, 2015). francs peuvent être adressés aux jeunes hommes afin de leur faire entendre les attentes normatives genrées, une empathie plus importante de la part des hommes est à noter. Elle serait alors peut-être à envisager comme une forme de solidarité masculine. Même si les aînés masculins font entendre leurs revendications et leurs espoirs en matière d'attentes sociales, cela expliquerait le fait qu'ils se montrent moins pressants. Il ne faut pas oublier que les injonctions qui touchent à la construction des identités masculines et de la virilité sont inscrites dans la reproduction de rapports de pouvoir (Bourdieu 2002).

C'est comme mon petit frère [Amar], lui, il a décidé de laisser les filles, parce qu'il est sportif et qu'il veut aller en Europe faire du sport. Les gens se moquent de lui et lui disent [...] qu'il aime pas les filles. Mais moi, quand on dit ça de lui, je dis merci ! Ça veut dire qu'il aime vraiment ce qu'il fait. (Ali, 2015)

Si des mécanismes et des rhétoriques de soutien masculines sont mises en place particulièrement par les plus jeunes, ils expriment également leur exaspération commune envers des attentes normatives genrées. Dès lors que l'on se réfère au cadre d'énonciation et de réitération de cet étiquetage, on peut apercevoir qu'il permet d'exprimer «de manière transposée les tensions et les conflits au sein de la communauté locale » (Bonhomme $2009: 15)$.

\section{Conclusion}

À travers cet article, j'ai tenté de montrer comment s'articulent, autour de la subjectivation de la mesure des désirs, les enjeux de la valorisation / dévalorisation de la réputation de goor gu bëgg jigeen. En jouissant d'une identité socialement positive, des jeunes hommes peuvent se dégager du « spectre de l'homosexualité » (Mulot 2009 : 119) et confirmer des capacités viriles. Tant que celles-ci ne portent pas atteinte aux valeurs et attentes normatives pesant sur le masculin, elles n'entachent pas directement 
l'aspect valorisant de cette réputation; c'est du moins l'hypothèse formulée suite à l'analyse des données recueillies. Puisqu'elle utilise des biais comparatifs (Fombrun 1996), cette réputation permettrait donc une double déconsidération sociale : celle de l'homme qui n'« aime pas les femmes», et celle de celui qui «ne les aime pas comme il faut ».

Cette rapide analyse a permis de mettre en lumière les mécanismes d'une injonction à l'hétérosexualité émanant d'un type d'institution précis, et elle a délaissé au passage d'autres sphères d'influence (religieuses, juridiques, etc.) qui agissent dans ce sens. Néanmoins, ce qui parait particulièrement intéressant tient à la force performative de cette réputation. Son énonciation et sa circulation, qui lui confèrent un statut de vérité, provoquent des réactions de toute part qui sont révélatrices d'un certain nombre d'enjeux familiaux, genrés et intergénérationnels présents dans la société sénégalaise. Les dynamiques décrites montrent alors que ce n'est pas l'injonction à l'hétéromasculinité à travers des assignations hétéronormatives que les hommes remettent en cause, mais les formes particulières qu'elle prend. L'analyse de cette réputation nous montre donc comment l'orientation sexuelle est associée à des normes qui dessinent les contours de ce qui relève du masculin, contribuant ainsi à (re)produire des rapports de genre et les relations hégémoniques qui leur sont associées.

\section{BIBLIOGRAPHIE}

ATTANÉ, Anne. 2009. « Quand la circulation de l'argent façonne les relations conjugales. L'exemple de milieux urbains au Burkina Faso » Autrepart 1 (49) : 155-171.

ANTOINE, Philippe, DJIRÉ, Mamadou \& NANITELAMIO, Jeanne. 1998. « Au cœur des relations hommes-femmes : polygamie et divorce » in Trois Générations de citadins au Sahel. Trente ans d'histoire sociale à Dakar et Bamako, ANTOINE, Philippe, OUEDRAOGO, Dieudonné \& PICHE, Victor (éd.). Paris : L'Harmattan, 147-180.

AUSTIN, John Langshaw. 1991 [1962]. Quand dire, c'est faire (G. Lane, trad.). Paris : Éditions du Seuil.

BAVA, Sophie. 2003. «De la "baraka aux affaires" : ethos économico-religieux et transnationalité chez les migrants Sénégalais mourides » Revue européenne des migrations internationales 19 (2) : 69-84.

BECKER, Howard. 1988 [1982]. Les Mondes de l'art (J. Bouniort, trad.). Paris : Flammarion.

BELLAH, Robert Neelly. 1986. "The Meaning of Reputation in American Society" California Law Review 3 (74) : 743-751.

BEUSCART, Jean-Samuel, CHAUVIN, Pierre-Marie, JOURDAIN, Anne \& NAULIN, Sidonie. 2015. « La Réputation et ses dispositifs » Terrains et travaux 1 (26) : 5-22.

BIAYA, Tshikala Kayembe. 2001. «Les Plaisirs de la ville : Masculinité, sexualité et féminité à Dakar (1997-2000) » African Studies Review 44 (2) : 71-85. 
BOISTEL, Philippe. 2014. « Réputation : un concept à définir » Communication et organisation 46 : 211-224.

BONHOMME, Julien. 2009. Les Voleurs de sexe. Anthropologie d'une rumeur africaine. Paris : Éditions du Seuil.

BOURDIEU, Pierre. 2002 [1998]. La Domination masculine. Paris : Éditions du Seuil.

BUTLER, Judith. 2005 [1990]. Trouble dans le genre. Le féminisme et la subversion de l'identité (C. Kraus, trad.). Paris : Éditions La Découverte.

CHAUVIN, Pierre-Marie. 2013. «La Sociologie des réputations. Une définition et cinq questions » Communications 2 (93) : 131-145.

CLAIR, Isabelle. 2008. Les Jeunes et l'amour dans les cités. Paris : Armand Colin.

DIMÉ, Mamadou Ndongo. 2007. « Remise en cause, reconfiguration ou recomposition ? Des solidarités familiales à l'épreuve de la précarité à Dakar » Sociologie et sociétés 39 (2) :151-171. DIOP, Abdoulaye-Bara. 2012 [1985]. La Famille wolof : tradition et changement. Paris : Karthala. ELSTER, Jon. 2013. « Réputation et caractère » Communications 2 (93) : 29-47.

EMLER, Nicholas. 2013. «La Réputation comme instrument social » Communications 2 (93) : 85-99.

FIDOLINI, Vulca. 2017. « Habiter l'ordre hétéronormatif et la masculinité par le mariage » Genre, sexualité \& société [En ligne], 17, consulté le 15 octobre 2017. URL : http://gss.revues.org/3993.

FOMBRUN, Charles. 1996. Reputation: Realizing Value from the Corporate Image. Boston : Harvard Business School Press.

FOUQUET, Thomas. 2014. «La Clandestinité comme stratégie. Sur la sexualité transactionnelle à Dakar » in L'échange économico-sexuel, BROQUA, Christophe \& DESCHAMPS, Catherine (éd.). Paris : Éditions EHESS, 125-152.

FREUD, Sigmund. 2012 [1905]. Trois Essais sur la théorie de la sexualité (M. Géraud, trad.). Paris : Éditions du Seuil.

GIAMI, Alain. 2000. «Les Récits sexuels : matériaux pour une anthropologie de la sexualité », Journal des anthropologues [En ligne], 82-83, consulté le 30 septembre 2016. URL : http:// jda.revues.org/3314.

GODELIER, Maurice. 2004. Les Métamorphoses de la parenté. Paris : Fayard.

GNING, Ndèye. 2013. «Les Motifs de l'illégitimité sociale de l'homosexualité au Sénégal » Africultures 6 (96) : 22-39.

HYMES, Dell. 1996. Ethnography, Linguistics, Narrative Inequality. Toward an Understanding of Voice. Londres : Taylor and Francis Ltd.

JOSEPH, Isaac. 1984. Le Passant considérable. Essai sur la dispersion de l'espace public. Paris : Librairie des méridiens.

LEIMDORFER, François. 2010. Les Sociologues et le langage. Paris : Maison des Sciences de l'Homme. NDOYE, Omar, SYLLA, Omar \& HOUNDECHANDJI, Edgar. 2003. « Impuissance interdite » in Le Sexe qui rend fou. Approches clinique et thérapeutique, NDOYE, Omar (éd.). Paris : Présence Africaine, 105-116.

MASQUELIER, Bertrand. 2005. «Anthropologie sociale et analyse du discours » Langage et société 4 (114) : 73-89. 
MULOT, Stéphanie. 2009. « Redevenir un homme en contexte antillais post-esclavagiste et matrifocal » Autrepart 1 (49) : 117-135.

ORIGGI, Gloria. 2013. «Présentation » Communications 2 (93) : 5-10.

PRONGER, Brian. 1990. The Arena of Masculinity. Sports, Homosexuality, and the Meaning of Sex. Londres : GMP Publishers.

SOHN, Anne-Marie. 2009. « Sois un Homme!» La construction de la masculinité au XIXe siècle. Paris : Éditions du Seuil.

SALOMON, Christine. 2009. « Antiquaires et businessmen de la Petite Côte du Sénégal. Le commerce des illusions amoureuses » Cahiers d'Études africaines 1 (193-194) : 147-173.

THORÉ, Luc. 1964. « Mariage et divorce dans la banlieue de Dakar » Cahiers d'Études africaines 4 (16) : 479-551.

VUARIN, Robert. 1990. «L'Enjeu de la misère pour l'islam sénégalais » Revue Tiers Monde 31 (123) : 601-621.

WACQUANT, Loïc. 2000. Corps et âme. Carnet ethnographique d'un apprenti boxeur. Marseille : Agone/ Comeau et Nadeau.

\section{NOTES}

1. Ce terme porte la focale sur les liens entre hétérosexualité et masculinité (Pronger 1990).

2. Cette thèse conduite à l'université Lumière Lyon 2 a reçu un financement (CDU) de la part de l'École doctorale 483.

3. Leur maison familiale située dans le quartier des Parcelles Assainies à Dakar est un espace domestique qui comprend, en fonction des circonstances et des mobilités de chacun-e-s, entre vingt et trente membres d'âges différents.

4. En particulier la maison familiale et les chambres louées par les deux hommes les plus âgés dont les parcours seront présentés.

5. Cinq à sept entretiens ont été réalisés avec chacun d'entre eux, espacés de quelques semaines à quelques mois.

6. L'aspect non objectif de la notion de réputation est une des raisons pour lesquelles son emploi, comme instrument mobilisable pour comprendre des données sociales, a longtemps été laissé de côté par les sciences humaines et sociales (Origgi 2013 : 6). La contribution de Becker a permis de revenir sur ce paradigme en montrant en quoi cette notion était à même d'éclairer des processus sociaux (1988).

7. Dans un souci d'anonymisation des acteurs de cette recherche, les noms ont été modifiés.

8. Afin de définir ce concept, je me réfère à la traduction qu'en donne C. Kraus : "Ce terme désigne le système, asymétrique et binaire, de genre, qui tolère deux et seulement deux sexes, où le genre concorde parfaitement avec le sexe (au genre masculin le sexe mâle, au genre féminin le sexe femelle) et où l'hétérosexualité (reproductive) est obligatoire, en tout cas désirable et convenable. » (Butler 2005 : 24)

9. Cette renonciation à tout contact sexuel pour les besoins des performances sportives est évoquée dans d'autres contextes, notamment celui de la boxe à Chicago (Wacquant $2000: 68$ ).

10. C'est un critère parmi d'autres, l'investissement économique d'un homme envers une femme est tout à fait récurrent comme manifestation d'attachement et de volonté d'engagement matrimonial (Fouquet 2014 ; Diop 2012).

11. Dans le cadre de cet article, seul le cas d'Amar est cité, mais j'ai pu observer à plusieurs reprises qu'un jeune homme qui se contenterait de regarder des jeunes femmes sans leur parler 
ni entretenir avec elles des relations sentimentales ne sera pas considéré comme «aimant les femmes ».

12. Les vignettes cliniques d'un groupe de recherche de la clinique psychiatrique de l'Hôpital Universitaire de Fann (Dakar) montrent clairement que, quel que soit l'âge d'un homme, ce dernier doit continuellement démontrer sa virilité et ainsi tout faire pour que des doutes sur sa force érectile ne soient pas émis (Ndoye et al 2003 : 114).

13. C. Salomon rapporte également cette idée à travers le fait qu' « un homme ne dit jamais non » (2009: 161).

14. Sachant que la notion de réputation renvoie nécessairement à un aspect relationnel (Bellah $1986: 743)$.

15. On retrouve ici la forme wolophisée du terme français "trop», qui est utilisé comme quantificateur du verbe « aimer ».

16. M. Dimé insiste grandement sur la solidarité familiale comme obligation sociale, tout en mettant en avant les recompositions qui marquent cette norme, notamment une plus grande implication des épouses pour aider leur mari dans leurs responsabilités domestiques (2007).

17. Sachant que la doctrine mouride à laquelle est affiliée cette famille met un accent tout particulier sur la valeur «travail» (Bava 2003).

18. Les règles de centralisation et de réciprocité dessinent les grands traits de ce communautarisme (Diop 2012 : 153).

19. Il faut préciser que le terme « tapette » est très souvent employé au Sénégal. S'il peut servir à désigner l'efféminement, la plupart du temps il est utilisé, par dérivation sémantique et indépendamment du sexe de la personne dont il est question, pour symboliser le fait d'avoir peur.

20. Nous parlons ici de ragots en insistant sur leur caractère localisé (Joseph 1984 : 39).

\section{RÉSUMÉS}

Cet article propose de s'intéresser aux manifestations discursives qui entourent les normes hétérosexuelles, lorsqu'elles émanent des membres d'une institution familiale sénégalaise pour être dirigées vers ses jeunes hommes. Pour ce faire, quatre profils d'hommes appartenant à la même unité familiale sont mobilisés afin d'interroger l'assignation de la réputation d' « aimer les femmes ». Nos observations montrent qu'il faut qu'un homme fasse preuve d'un certain nombre d'aptitudes afin d'être considéré comme "aimant les femmes ». Ses capacités en termes de séduction sont mises à l'épreuve tout autant que celles visant à entretenir des relations sentimentales avec plusieurs partenaires. Cette assignation est valorisante pour les jeunes hommes dakarois, jusqu'à un certain point. Il ne faudrait pas que la réputation d' «aimer les femmes" les empêche d'accéder à la reconnaissance sociale obtenue par une alliance matrimoniale. Ainsi pour eux, il ne faudrait pas «trop aimer les femmes » ou les « aimer trop longtemps", sous peine de produire une stigmatisation excessive. Cet article tend à concevoir la notion de réputation comme un énoncé de jugement, porté par un groupe sur l'un de ses membres. Si c'est un outil permettant de faire entendre des aspirations hétéronormatives, il s'avère intéressant de questionner les manières dont s'articulent autour de la subjectivation de la mesure des désirs masculins, les enjeux de la valorisation/dévalorisation d'une réputation genrée. Si cette réputation énonce clairement une injonction sociale de virilité et d'hétérosexualité, elle exprime également les limites de son fonctionnement en termes de 
reproduction sociale lorsque les individus qui en sont porteurs mettent en place des stratégies de résistance et de contournement.

This article examines the discursive manifestations around heterosexual norms, when they emanate from members of the Senegalese family institution to young men. To do so, we will present four different male profiles, all belonging to the same family unit, in order to understand how someone comes to be designated by the reputation of "loving women". Our observations show that a man must display a certain number of aptitudes in order to be considered as "loving women". His seduction capabilities are tested as well as his ability to sustain multiple romantic relationships. This reputation is gratifying for young men from Dakar, up to a point. Their reputation of "loving women" shouldn't prevent them from reaching a social recognition obtained through matrimonial alliance. Thus, they shouldn't "love women too much" or "love them too long", or they would risk an excessive stigmatization. This article tends to conceive of the notion of reputation as the wording of judgements by a group, on one of its member. If this is a tool enabling normative and heteronormative aspirations to be heard, it is interesting to question the various issues of appreciation/depreciation of a gendered reputation, centered around the subjectivization of the evaluation of masculine desires. If this reputation clearly articulates a social injunction to virility and heterosexuality, it also expresses the limits of its functioning in terms of social reproduction when the individuals bearing it develop strategies of resistance and of getting around it.

\section{INDEX}

Thèmes : Recherches

Keywords : reputation, virility, seduction, family, socialisation

Mots-clés : réputation, virilité, séduction, famille, socialisation

\section{AUTEUR}

\section{NICOLAS FAYNOT}

LADEC - Fre2002

Université Lyon 2 - ENS de Lyon - CNRS

Nicolas Faynot est doctorant en anthropologie à l'université Lumière Lyon 2. Dans son travail de thèse, il s'intéresse principalement aux relations sentimentales/sexuelles de la jeunesse sénégalaise, à la circulation monétaire dans ces relations et à la construction des masculinités. 